\title{
An Optimization Problem in Deregulated Electricity Markets solved with the Nonsmooth Maximum Principle
}

\author{
L. Bayón ${ }^{1}$, J.M. Grau ${ }^{1}$, M.M. Ruiz ${ }^{1}$ and P.M. Suárez ${ }^{1}$ \\ ${ }^{1}$ Department of Mathematics, University of Oviedo, Spain \\ emails: bayon@uniovi.es, grau@uniovi.es, mruiz@uniovi.es, pedrosr@uniovi.es
}

\begin{abstract}
In this paper, the new short-term problems that are faced by a generation company in a deregulated electricity market are addressed and a optimization algorithm is proposed. Our model of the spot market explicitly represents the price of electricity as an uncertain exogenous variable. We consider a very complex problem of hydrothermal optimization with pumped-storage plants, so the problem deals with non-regular Lagrangian and non-holonomic inequality constraints. To obtain a necessary minimum condition, the problem was formulated within the framework of nonsmooth analysis using the generalized (or Clarke's) gradient and the Nonsmooth Maximum Principle. The optimal control problem is solved by means of an algorithm implemented in the commercial software package Mathematica. Results of the application of the method to a numerical example are presented.
\end{abstract}

Key words: Nonsmooth Analysis, Control Problem, Electricity markets

MSC 2000: 49M20, 49J24, 91B24, 91B26

\section{Introduction}

Over the last decade, the electricity industry has experienced significant changes in terms of deregulation and competition. In this paper, we focus on the problem that a generation company faces when preparing its offers for the day-ahead market. Several methods have been proposed for simulating competitive generation markets. Most of these models [1] can be categorized into two major groups: models that represent all the generation companies and models that focus on a particular generation company. Two approaches can be adopted to represent spot market auctions when only one company is considered: price modeled as an exogenous variable and price modeled as a function of the demand supplied by the firm under study. In the former, the price of electricity does not depend on the company's decisions. This can be acceptable if the company is small enough. These models can again be classified into two sub-groups, depending on whether they use a deterministic [2] or probabilistic [3] price representation. 
In this paper, we only represent the operation of one company in detail, including each of the company's generation units. Our model of the spot market explicitly represents the price of electricity as an uncertain exogenous variable. We represent generation units at a high level of detail and our model distinguishes individual generation units and considers inter-temporal constraints such as hydro reserves. In addition, we also consider pumped-storage hydro-plants.

The Spanish activity rules [4] have been used as a reference model for the market. The day-ahead market in the Spanish wholesale electricity market is organized as a set of twenty-four simultaneous hourly auctions. The simple bid format consists of a pair of (hourly) values: quantity $q[M W h]$ and price $p[$ euro $/ M W h]$. The utility company that inspires our paper, $H C$, controls approximately only $7 \%$ of all the electricity that is generated. So, we consider our company as a price-taker, and under this assumption, the volatility of the spot market price of electricity is represented by a stochastic model. Price forecasting techniques in power systems are relatively recent procedures [5] [6]. Although the problem of constructing the probability distribution exceeds the purpose of this paper, we suggest the following simplified approach based on [7]. The idea is to search for past spot market sessions that can be considered similar to the session that the company is about to face. To identify the days, we classify the entire collection of sessions (using clustering techniques) according to the values of an explanatory variable. The most relevant information about the current session for our problem is the vector of 24 prices that has resulted from the day-ahead market clearing. Once a group of $S$ similar days has been identified, the company can assume that the probability distribution for the market session under study is completely defined by these past $S$ market sessions (probability distributions with finite support). If we now focus on a particular auction, it is easy to understand that the $S$ quantities and $S$ prices decided by the company for that hour constitute the offer curve (nondecreasing) that the company must submit to that auction.

This paper addresses a very complex problem of hydrothermal optimization with pumped-storage plants. In this kind of problem (see the previous paper [8]), the Lagrangian is piecewise continuous and we consider constraints for the admissible generated power. Hence, this paper considers non-regular Lagrangian and non-holonomic inequality constraints (differential inclusions). To obtain a necessary minimum condition, the problem is formulated within the framework of nonsmooth analysis [9] using the generalized (or Clarke's) gradient and the Nonsmooth Maximum Principle. This characteristic distinguishes our work from all the above.

\section{Statement of the Problem}

In this section the optimization problem of one company is described, the objective function of which can be defined as its profit maximization. Let us assume that our hydrothermal system accounts for $n$ hydro-plants and $m$ thermal plants: the $\left(H_{\mathbf{n}}-T_{\mathbf{m}}\right)$ problem.

Let $\Psi_{i}: D_{i} \subseteq \mathbb{R}^{+} \longrightarrow \mathbb{R}^{+}(i=1, \ldots, m)$ be the cost functions (euro/h) of the $m$ 
thermal plants. The most usual cost function of each generator can be represented as a quadratic function:

$$
\Psi_{i}\left(P_{i}(t)\right)=\alpha_{i}+\beta_{i} P_{i}(t)+\gamma_{i} P_{i}^{2}(t) ; \quad i=1, \ldots, m
$$

where $P_{i}(M W)$ is the power generated, and we consider the thermal plants to be constrained by technical restrictions of the type

$$
P_{i \min } \leq P_{i}(t) \leq P_{i \max } ; \quad i=1, \ldots, m, \forall t \in[0, T]
$$

$[0, T]$ being the optimization interval. In prior studies [10], it was proven that the problem with $m$ thermal plants may be reduced to the study of a hydrothermal system made up of one single thermal plant, called the thermal equivalent: the $\left(H_{\mathbf{n}}-T_{\mathbf{1}}\right)$ problem. We shall denote as the equivalent minimizer of $\left\{\Psi_{i}\right\}_{1}^{m}$, the function $\Psi$ : $D_{1}+\cdots+D_{m} \rightarrow \mathbb{R}$ defined by

$$
\Psi(P(t))=\min \sum_{i=1}^{m} \Psi_{i}\left(P_{i}(t)\right) ; P_{\min } \leq P(t) \leq P_{\max }
$$

with $P(t)$ the power generated by said thermal equivalent.

We assume that our system accounts for $n$ hydro-plants that have a pumping capacity. The mapping $H: \Omega_{H} \longrightarrow \mathbb{R}$

$$
H\left(t, z_{1}(t), \ldots, z_{i}(t), \ldots, z_{n}(t), \dot{z}_{1}(t), \ldots, \dot{z}_{i}(t), \ldots, \dot{z}_{n}(t)\right)=H(t, \mathbf{z}(t), \dot{\mathbf{z}}(t))
$$

is called the function of effective hydraulic contribution and is the power contributed to the system at the instant $t$ by the set of hydro-plants, $z_{i}(t)$ being the volume that is discharged up to the instant $t$ by the $i$-th hydro-plant, $\dot{z}_{i}(t)$ the rate of water discharge at the instant $t$ by the $i$-th hydro-plant, and $\Omega_{H} \subset[0, T] \times \mathbb{R}^{n} \times \mathbb{R}^{n}$ the domain of definition of $H$.

We say that $\dot{\mathbf{z}}=\left(z_{1}, \ldots, z_{n}\right)$ is admissible for $H$ if $z_{i}$ belong to the class $\widehat{C}^{1}[0, T]$ (the set of piecewise $C^{1}$ functions), and $(t, \mathbf{z}(t), \dot{\mathbf{z}}(t)) \in \Omega_{H}, \forall t \in[0, T]$. The volume $b_{i}$ that must be discharged up to the instant $T$ is called the admissible volume of the $i$-th hydro-plant. Let $\mathbf{b}=\left(b_{1}, \ldots, b_{n}\right) \in \mathbb{R}^{n}$ be the vector of admissible volumes. In a general model, with hydraulic coupling between the $n$ hydro-plants, we call $H_{i}\left(t, z_{i}(t), \dot{z}_{i}(t)\right)$ : $\Omega_{H_{i}}=[0, T] \times \mathbb{R} \times \mathbb{R} \longrightarrow \mathbb{R}$ the function of effective hydraulic contribution by the $i$-th hydro-plant, being

$$
H(t, \mathbf{z}(t), \dot{\mathbf{z}}(t))=\sum_{i=1}^{n} H_{i}\left(t, z_{i}(t), \dot{z}_{i}(t)\right)
$$

Besides, we consider $H_{i}\left(t, z_{i}(t), \dot{z}_{i}(t)\right)$ to be bounded by technical constraints

$$
H_{i \min } \leq H_{i}\left(t, z_{i}(t), \dot{z}_{i}(t)\right) \leq H_{i \max } ; i=1, \cdots, n, \forall t \in[0, T]
$$

Throughout the paper, no transmission losses will be considered; a crucial aspect when addressing the optimization problem from a centralized viewpoint. From the perspective of a generation company, and within the framework of the new electricity 
market, said losses are not relevant, since the generators currently receive payment for all the energy they generate in power plant bars.

Let us assume that the cost function $\Psi: \mathbb{R}^{+} \longrightarrow \mathbb{R}^{+}$satisfies $\Psi^{\prime}(x)>0, \forall x \in \mathbb{R}^{+}$, i.e. it is strictly increasing. This constraint is absolutely natural: it reads more cost to more generated power. Let us assume as well that $\Psi^{\prime \prime}(x)>0, \forall x \in \mathbb{R}^{+}$, i.e. it is strictly convex. The models traditionally employed meet this constraint.

Let us assume that the function $H_{i}$ is strictly increasing with respect to the rate of water discharge $\dot{z}_{i}$, i.e. $\partial H_{i} / \partial \dot{z}_{i}>0$ (more power to a higher rate of water discharge) and that $\left[\partial H_{i} / \partial z_{i}\right]_{\dot{z}_{i}=0}=0$. Let us also assume that $\partial^{2} H_{i} / \partial \dot{z}_{i}^{2}<0$, i.e. $H_{i}$ is concave with respect to $\dot{z}_{i}$. The real models meet these three constraints. In addition, pumpedstorage plants are considered, and in this kind of problem, the derivative of $H_{i}$ with respect to $\dot{z}_{i}\left(\partial H_{i} / \partial \dot{z}_{i}\right)$ presents discontinuity at $\dot{z}_{i}=0$, which is the border between the power generation zone (positive values of $\dot{z}_{i}$ ) and the pumping zone (negative values of $\left.\dot{z}_{i}\right)$. In the real models, it is verified that $H_{\dot{z}}^{+} \leq H_{\dot{z}}^{-}$. In the $\left(H_{\mathbf{n}}-T_{\mathbf{1}}\right)$ problem, the objective function is given by revenue minus cost during the optimization interval $[0, T]$

$$
F(P, \mathbf{z})=\int_{0}^{T}[p(t)(P(t)+H(t, \mathbf{z}(t), \dot{\mathbf{z}}(t)))-\Psi(P(t))] d t
$$

Revenue is obtained by multiplying the total production (thermal and hydraulic) of the company by the clearing price $p(t)$ in each hour $t$. Cost is given by $\Psi$, the cost function of the thermal equivalent, where $P(t)$ is the power generated by said plant. With this statement, our objective functional in continuous time form is

$$
\max _{P, \mathbf{z}} F(P, \mathbf{z})=\max _{P, \mathbf{z}} \int_{0}^{T} L(t, P(t), \mathbf{z}(t), \dot{\mathbf{z}}(t)) d t
$$

with $L(t, P(t), \mathbf{z}(t), \dot{\mathbf{z}}(t))=p(t)(P(t)+H(t, \mathbf{z}(t), \dot{\mathbf{z}}(t)))-\Psi(P(t))$, on the set

$$
\Omega=\left\{\mathbf{z} \in\left(\widehat{C}^{1}[0, T]\right)^{n} \mid \begin{array}{c}
z_{i}(0)=0, z_{i}(T)=b_{i} \\
H_{i \min } \leq H_{i}\left(t, z_{i}(t), \dot{z}_{i}(t)\right) \leq H_{i \max }, \quad \forall t \in[0, T] \\
\forall i=1, \ldots, n
\end{array}\right\}
$$

\section{The $\left(H_{1}-T_{1}\right)$ Problem}

We begin the development in this section by presenting the simple problem with one pumped-storage hydro-plant $(i=1)$. In the $\left(H_{\mathbf{1}}-T_{\mathbf{1}}\right)$ problem, we have $\mathbf{z}=z$ and our objective functional is

$$
F(P, z)=\int_{0}^{T} L(t, P(t), z(t), \dot{z}(t)) d t
$$

with $L(t, P(t), z(t), \dot{z}(t))=p(t)(P(t)+H(t, z(t), \dot{z}(t)))-\Psi(P(t))$ on the set

$$
\Omega=\left\{z \in \widehat{C}^{1}[0, T] \mid \quad \begin{array}{c}
z(0)=0, z(T)=b \\
H_{\min } \leq H(t, z(t), \dot{z}(t)) \leq H_{\max }, \quad \forall t \in[0, T]
\end{array}\right\}
$$


where $L(\cdot, \cdot, \cdot, \cdot)$ and $L_{z}(\cdot, \cdot, \cdot, \cdot)$ are the class $C^{0}$ and $L_{\dot{z}}(t, P, z, \cdot)$ is piecewise continuous $\left(L_{\dot{z}}(t, P, z, \cdot)\right.$ is discontinuous in $\left.\dot{z}=0\right)$. The problem involves non-holonomic inequality constraints (differential inclusions) and the previous assumptions guarantee that: $L_{\dot{z} \dot{z}}(t, P, z, \dot{z})<0 ; L_{\dot{z}}(t, P, z, \dot{z})>0$. We also assume that

$$
H(t, b, \dot{z}(t)) \leq H(t, z(t), \dot{z}(t)) \leq H(t, 0, \dot{z}(t)), \forall z \in \Omega
$$

These suppositions are fulfilled in all real hydrothermal problems, and bearing in mind the weak influence of $z(t),(H(t, b, \dot{z}) \simeq H(t, z, \dot{z}) \simeq H(t, 0, \dot{z}))$, it is reasonable to substitute the restriction: $H_{\min } \leq H(t, z(t), \dot{z}(t)) \leq H_{\max }$ by others of the type: $H_{\min } \leq$ $H(t, b, \dot{z}) ; H(t, 0, \dot{z}) \leq H_{\max }$. Thus, it is reasonable to substitute $\Omega$ by

$$
\Omega^{*}=\left\{z \in \widehat{C}^{1}[0, T] \mid \quad \begin{array}{c}
z(0)=0, z(T)=b \\
H_{\min } \leq H(t, b, \dot{z}) ; H(t, 0, \dot{z}) \leq H_{\max }, \forall t \in[0, T]
\end{array}\right\}
$$

The solution to the problem in $\Omega^{*}$ will be very close to that obtained with the set $\Omega$, the advantage being that the mathematical treatment of sets of type $\Omega^{*}$ is much simpler than of those of type $\Omega$. We shall focus in the present paper on the development of the applications of Optimal Control Theory (OCT) and nonsmooth analysis to this problem. Let us term as the coordination function of $z \in \Omega^{*}$ the function in $[0, T]$, defined by:

$$
\mathbb{Y}_{z}(t)=L_{\dot{z}}(t, P(t), z(t), \dot{z}(t))-\int_{0}^{t} L_{z}(s, P(s), z(s), \dot{z}(s)) d s
$$

denoting by $\mathbb{Y}_{z}^{+}(t)$ and $\mathbb{Y}_{z}^{-}(t)$ the expressions obtained when considering the lateral derivatives of $L$ with respect to $\dot{z}$. Let us now see the fundamental result, which is the basis for elaborating the optimization algorithm. We present the problem considering the state variables to be $z(t)$ and $P(t)$ and the control variables $u_{1}(t)=\dot{z}(t)$ and $u_{2}(t)=\dot{P}(t)$. The optimal control problem is thus:

$$
\begin{gathered}
\max _{u_{1}(t), u_{2}(t)} \int_{0}^{T} L\left(t, P(t), z(t), u_{1}(t)\right) d t ; \quad \text { with }\left\{\begin{array}{l}
\dot{z}=u_{1} ; \quad \dot{P}=u_{2} \\
z(0)=0, z(T)=b
\end{array}\right. \\
u_{1}(t) \in \Theta=\left\{x \mid H_{\min } \leq H(t, b, x) ; H(t, 0, x) \leq H_{\max }\right\} ; u_{2}(t) \in(-\infty, \infty)
\end{gathered}
$$

We shall use the nonsmooth version of Pontryagin's Minimum Principle (PMP) [9] as the basis for proving this theorem.

Theorem 1 (Theorem of Coordination). If $\left(z^{*}, P^{*}\right) \in\left(\widehat{C}^{1}, C^{1}\right)$ is a solution of our problem, then $\exists K \in \mathbb{R}^{+}$such that:

$$
\begin{aligned}
& \text { i) If } \dot{z}^{*}(t)=0 \Longrightarrow \mathbb{Y}_{z^{*}}^{+}(t) \leq K \leq \mathbb{Y}_{z^{*}}^{-}(t) \\
& \text { ii) If } \dot{z}^{*}(t) \neq 0 \Longrightarrow \mathbb{Y}_{z^{*}}(t) \text { is }\left\{\begin{array}{lll}
\geq K & \text { if } & H\left(t, b, \dot{z}^{*}(t)\right)=H_{\min } \\
=K & \text { if } & H_{\min }<H\left(t, z^{*}(t), \dot{z}^{*}(t)\right)<H_{\max } \\
\leq K & \text { if } & H\left(t, 0, \dot{z}^{*}(t)\right)=H_{\max }
\end{array}\right. \\
& \text { and } \quad \dot{\Psi}\left(P^{*}(t)\right)=p(t)
\end{aligned}
$$

We shall call this relation

$$
L_{\dot{z}}(t, P(t), z(t), \dot{z}(t))-\int_{0}^{t} L_{z}(s, P(s), z(s), \dot{z}(s)) d s=K \in \mathbb{R}^{+}, \forall t \in[0, T]
$$


the coordination equation for $z(t)$, and the positive constant $K$ will be termed the coordination constant of the extremal.

Note. It is very important to stress that the problem is thus easily broken down into the two sub-problems: Thermal and Hydro. In the thermal sub-problem, the power $P(t)$ of the equivalent thermal plant is distributed (as we see in [10]) between the $m$ thermal plants, and so is completely resolved. In the next section, we consider once more the general problem $\left(H_{\mathbf{n}}-T_{\mathbf{1}}\right)$ with $n$ hydro-plants, which is the problem to be solved.

\section{Generalization to the $\left(H_{\mathrm{n}}-T_{1}\right)$ Problem. The Optimization Algorithm}

In this section, we present an algorithm of the numerical resolution of the problem of optimization of a hydrothermal system that involves $n$ hydro-plants. The associated variational problem is related to solving a boundary-value problem for a system of differential equations. The algorithm uses a particular strategy related to the method of cyclic coordinate descent (CCD). The CCD method minimizes a function cyclically with respect to the coordinate variables. With our method, a problem of the type $H_{\mathbf{n}}-T_{\mathbf{1}}$ could be solved (under certain conditions) if we start out from the resolution of a sequence of problems of the type $H_{\mathbf{1}}-T_{\mathbf{1}}$. The algorithm for the $H_{\mathbf{n}}-T_{\mathbf{1}}$ problem carries out several iterations and at each $j$-th iteration calculates $n$ stages, one for each hydro-plant. At each stage, it calculates the optimal functioning of a hydro-plant, while the behavior of the rest of the plants is assumed fixed. For every $\mathbf{z}=\left(z_{1}, \ldots, z_{n}\right) \in \Omega$, we consider the functional $F_{\mathbf{z}}^{i}$ defined by

$$
\begin{aligned}
F_{\mathbf{z}}^{i}\left(P, v_{i}\right) & =\int_{0}^{T}\left[p(t)\left(P(t)+H_{\mathbf{z}}^{i}\left(t, v_{i}(t), \dot{v}_{i}(t)\right)\right)-\Psi(P(t))\right] d t \\
\text { with } H_{\mathbf{z}}^{i}\left(t, v_{i}, \dot{v}_{i}\right) & =H\left(t, z_{1}, \ldots, z_{i-1}, v_{i}, z_{i+1}, \ldots, z_{n}, \dot{z}_{1}, \ldots, \dot{z}_{i-1}, \dot{v}_{i}, \dot{z}_{i+1}, \ldots, \dot{z}_{n}\right)
\end{aligned}
$$

where $H_{\mathbf{z}}^{i}$ represents the power generated by the hydraulic system as a function of the rate of water discharge and the volume turbinated by the $i$-th plant, under the assumption that the rest of the plants behave in a definite way. We call the $i$-th minimizing mapping the mapping $\phi_{i}: \Omega \longrightarrow \Omega$, defined in the following way: for every $\mathbf{z} \in \Omega$

$$
\phi_{i}\left(P, z_{1}, \ldots, z_{i}, \ldots, z_{n}\right)=\left(P^{*}, z_{1}, \ldots, z_{i}^{*}, \ldots, z_{n}\right)
$$

where $\left(P^{*}, z_{i}^{*}\right)$ minimizes $F_{\mathbf{z}}^{i}$. If we set $\Phi=\left(\phi_{n} \circ \phi_{n-1} \circ \cdots \circ \phi_{2} \circ \phi_{1}\right)$ and

$$
\left(P^{j}, \mathbf{z}^{j}\right)=\Phi\left(P^{j-1}, \mathbf{z}^{j-1}\right)
$$

beginning with some admissible $\left(P^{0}, \mathbf{z}^{0}\right)$, we construct a sequence of $\left(P^{j}, \mathbf{z}^{j}\right)$ via successive applications of $\left\{\phi_{i}\right\}_{i=1}^{n}$ and the algorithm will search

$$
\lim _{j \rightarrow \infty}\left(P^{j}, \mathbf{z}^{j}\right)
$$


It is simple to justify the convergence of the algorithm in a finite number of steps, simply by considering the following solution set:

$$
\{\mathbf{z} \mid F(P, \mathbf{z})-F(\Phi(P, \mathbf{z}))<\varepsilon\}
$$

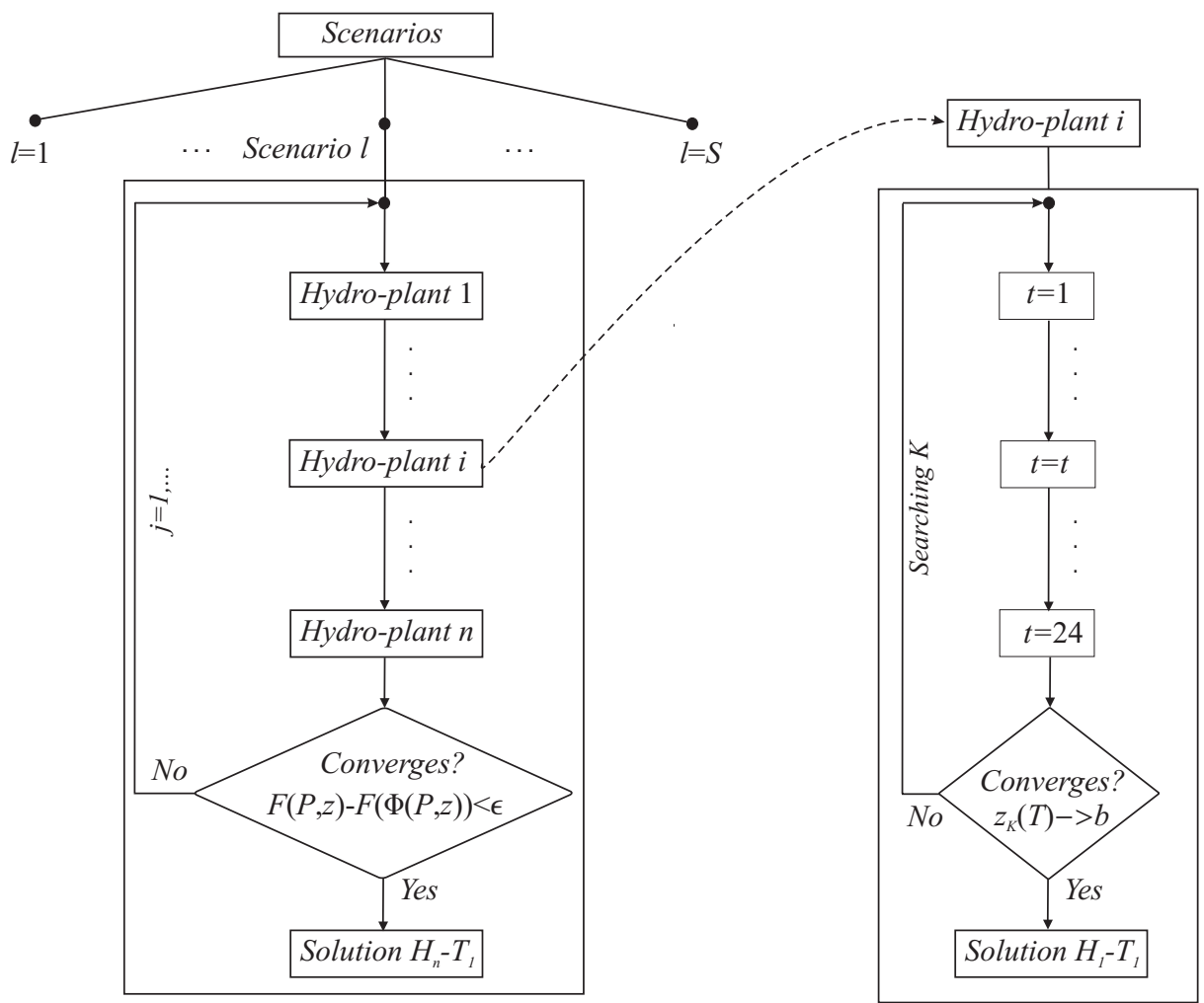

Fig. 1. The Optimization Algorithm.

The application of every $\phi_{i}$ involves solving a problem of the type $\left(H_{1}-T_{1}\right)$. To obtain the optimum operating conditions of the hydro-plant, we shall use the coordination equation (3.1). To undertake the approximate calculation of the solution, expressed in Theorem 1, we use a similar numerical method to those used to solve differential equations in combination with an appropriate adaptation of the classical shooting method.

Step 1) Approximate construction of $z_{K}$ (the adapted Euler method).

The problem will consist in finding for each $K$ the function $z_{K}$ that satisfies $z_{K}(0)=0$, and the conditions of Theorem 1. From the computational point of view, the construction of $z_{K}$ can be performed with the use of a discretized version of Equation (3.1). The approximate construction of each $z_{K}$, which we shall call $\widetilde{z}_{K}$, is carried out by means of polygonals (Euler's method). In general, the construction of $\dot{z}_{K}$ must be carried out by constructing and successively concatenating the extremal arcs and boundary arcs until completing the interval $[0, T]$.

Step 2) Construction of a sequence $\left\{K_{j}\right\}_{j \in \mathbb{N}}$ such that $z_{K_{j}}(T)$ converges to $b$ (the adapted shooting method). 
Varying the coordination constant $K$, we would search for the extremal that fulfils the second boundary condition $z_{K}(T)=b$. The procedure is similar to the shooting method used to resolve a two-point boundary value problem (TPBVP). A number of methods exist for solving these problems, including shooting, collocation and finite difference methods. Among the shooting methods, the Simple Shooting Method (SSM) and the Multiple Shooting Method (MSM) appear to be the most widely known and used methods. We implemented a SSM and obtained good results. Effectively, we may consider the function $\varphi(K):=z_{K}(T)$ and calculate the root of $\varphi(K)-b=0$, which may be realized approximately using elemental procedures. The secant method was used in the present paper, and the algorithm shows a rapid convergence to the optimal solution for a wide range of $K_{\min }$ and $K_{\max }$.

\section{Application to a Real Hydrothermal System}

A computer program was written (using the Mathematica package) to apply the results obtained in this paper to a real power system. As an example, we shall use the hydrothermal system that the electricity company $H C$ has in Asturias (Spain), which is made up of 2 classic thermal plants: Aboño (with two groups of 360 and 543 of power $(M w)$ respectively) and Soto (with two groups of 254 and 350 of power $(M w)$ respectively) and 9 hydro-plants. For our optimization problem, we shall only use the 3 variable-head (the generation is function of $z$ and $\dot{z}$ ) hydro-plants of the utility company $H C$ : Salime, Tanes (pumped-storage) and La Barca. We do not consider the remaining hydro-plants, because they are run-of-river type (without reservoir) and power generation is not controllable. Let us see the models of different subsystems used in our study. For the cost functions, we use a second-order polynomial

$$
\Psi_{i}\left(P_{i}(t)\right)=\alpha_{i}+\beta_{i} P_{i}(t)+\gamma_{i} P_{i}^{2}(t)
$$

The hydro-network has the three hydro-plants on different rivers, so the rate of discharge at the upstream plant does not affect the behaviour at the downstream plants: the hydraulic system has no hydraulic coupling. We use a variable head model and the $i$-th function of effective hydraulic generation $H_{i}$ (for a conventional hydro-plant) is given by

$$
H_{i}\left(t, z_{i}(t), \dot{z}_{i}(t)\right)=A_{i}(t) \dot{z}_{i}(t)-B_{i} \dot{z}_{i}(t) z_{i}(t)-C_{i} \dot{z}_{i}^{2}(t) ; \quad \dot{z}_{i}(t) \geq 0
$$

where $A_{i}(t), B_{i}$ and $C_{i}$ are the coefficients

$$
A_{i}(t)=\frac{1}{G_{i}} B_{y_{i}}\left(S_{0 i}+t \cdot i_{i}\right) ; \quad B_{i}=\frac{B_{y_{i}}}{G_{i}} ; \quad C_{i}=\frac{B_{t_{i}}}{G_{i}}
$$

For the pumped-storage plant, $H_{i}$ is defined piecewise, taking in the pumping zone $\left(\dot{z}_{i}(t)<0\right): M \cdot H_{i}\left(t, z_{i}(t), \dot{z}_{i}(t)\right)$. The parameters that appear in this formula are: the efficiency $G$ in $\left(m^{4} / h . M w\right)$, the natural inflow $i$ in $\left(\mathrm{m}^{3} / h\right)$, the initial volume $S_{0}$ in $\left(10^{6} \mathrm{~m}^{3}\right)$, and the coefficients $B_{y}$ in $\left(10^{-7} \mathrm{~m}^{-2}\right)$ and $B_{t}$ in $\left(10^{-5} \mathrm{hm}^{-2}\right)$, parameters that depend on the geometry of the reservoir. 
Let us consider the construction of the scenario structure for the day-ahead market problem faced by the company $H C$ in the Spanish spot market. In particular, the market session of February 15th 2006 is considered as the current session. The past market sessions [4] that are considered relevant range from February 1st to February 14th. Table I presents the results of the clustering analysis performed on this range of days. The classification provided by the $S$-means algorithm for $S=4$ (four clusters) is presented below.

Table I. Clustering Analysis.

\begin{tabular}{|c|c|c|c|ccc|c|c|c|c|ccc|c||c||}
\hline Date & 1 & 2 & 3 & 4 & 5 & 6 & 7 & 8 & 9 & 10 & 11 & 12 & 13 & 14 & 15 \\
\hline Day & $\mathrm{W}$ & $\mathrm{Th}$ & $\mathrm{F}$ & $\mathrm{Sa}$ & $\mathrm{S}$ & $\mathrm{M}$ & $\mathrm{T}$ & $\mathrm{W}$ & $\mathrm{Th}$ & $\mathrm{F}$ & $\mathrm{Sa}$ & $\mathrm{S}$ & $\mathrm{M}$ & $\mathrm{T}$ & $\mathrm{W}$ \\
\hline Cluster & 4 & 4 & 4 & 1 & 2 & 3 & 4 & 4 & 4 & 4 & 1 & 2 & 3 & 4 & 4 \\
\hline
\end{tabular}

As can be seen, the four day types provided by the clustering analysis are quite reasonable: Cluster 1 and Cluster 2 corresponds to low-price days (Saturdays and Sundays, respectively), Cluster 3 includes one type of weekday: Mondays, and Cluster 4 comprises the other type of weekdays. This analysis suggests considering eight scenarios (eight realizations) for the day-ahead market problem faced by the company on February 15th. We consider short-term hydrothermal scheduling (24 hours) with an optimization interval $[0,24]$ and we consider a discretization of 24 subintervals. The total optimal hydro and thermal power generation for the company $\mathrm{HC}$ are shown in Figure 2-a and Figure 2-b respectively. The eight scenarios considered are presented in both figures.

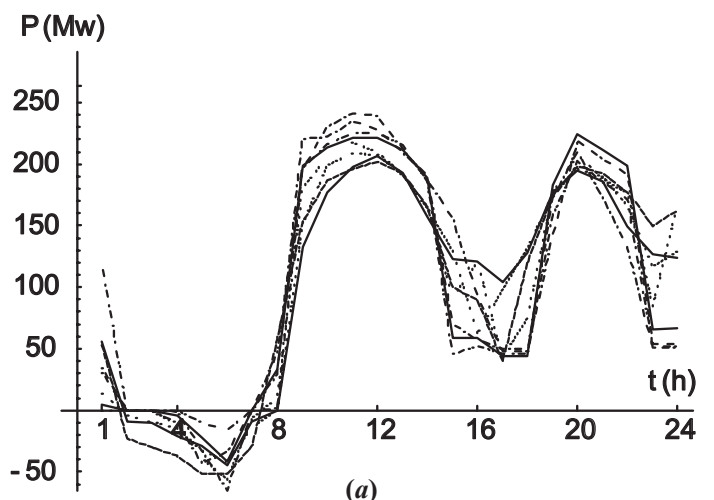

Fig. 2. (a) Optimal hydro-power. (b) Optimal thermal-power.

The solution yields the optimal offers that the company must submit to each of the dayahead market auctions. Figure 3-a shows the offers corresponding to the 4th auction for the total optimal thermal-power, and for the eight possible realizations. The 8 quantities and 8 prices for that hour constitute the offer curve (nondecreasing) that the 
company must submit to that auction.

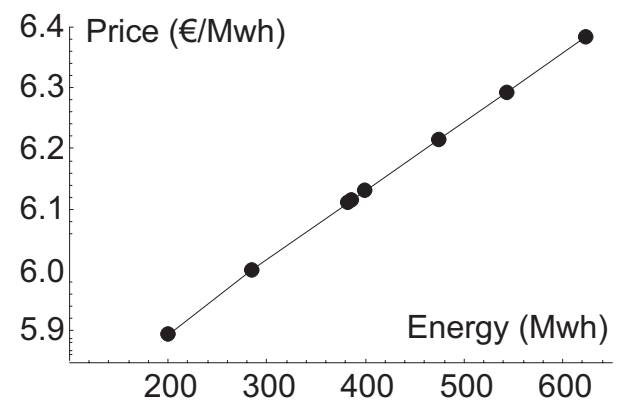

(a)

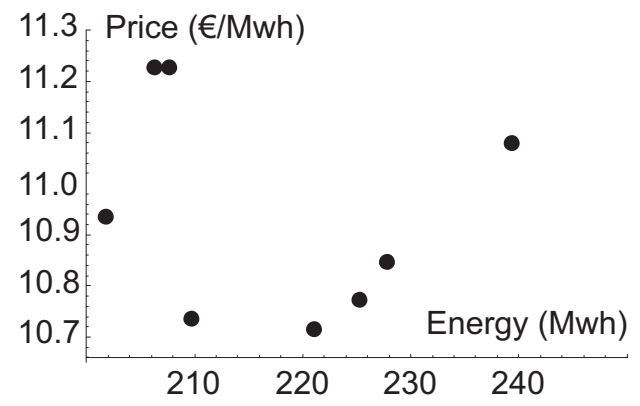

(b)

Fig. 3. (a) Thermal-offers. (b) Hydro-offers.

These results can be easily analyzed. Figure 3-a shows that the offer curve obtained for the 4th hourly auction is quite flat, thus making the company rather uncertain about the amount of energy that it will finally sell. This is confirmed by Figure 2-b, in which the company's eight possible levels of sales for the 4th hour are very different. However, it is not possible to construct an offer curve (nondecreasing) for the company's optimal hydro-power. Figure 3-b shows the offers corresponding to the 12th auction for the total optimal hydro-power, and for the eight possible realizations. It is easy to understand that this behaviour is due to the inter-temporal constraints for the hydroplants, besides the pumped-storage character of some of the hydro-plants (the optimal hydro-solution of one of the auctions influences the rest of the auctions). Therefore, we suggest that the optimal offers that the company must submit, for the hydro-plants, must be a half value of the optimal hydro-power generation that we present in Figure 2 -a.

\section{References}

[1] M. Ventosa, A. Baíllo, M. Rivier and A. Ramos, Electricity Market Modeling Trends, Energy Policy 33(7) (2005) 897-913.

[2] G. Gross and D.J. Finlay, Optimal Bidding Strategies in Competitive Electricity Markets, Proc. 12th PSCC (1996) 815-823.

[3] J. Valenzuela and M. Mazumdar, Probabilistic Unit Commitment under a Deregulated Market, in: B. F. Hobbs, M. H. Rothkopf, R. P. O'Neill and H.-P. Chao, (Eds.), The Next Generation of Electric Power Unit Commitment Models, Kluwer Academic Publishers, Boston, (2001) 139-152.

[4] Compañía Operadora del Mercado Español de Electricidad, S.A. Electricity market activity rules. http://www.omel.es/es/pdfs/EMRules.pdf.

[5] A.J. Conejo, F.J. Nogales and J.M. Arroyo, Price-taker bidding strategy under price uncertainty, IEEE Trans. Power Sys. 17(4) (2002) 1081-1088. 
[6] A. Mateo, A. MuÑoz And J. García, Modeling and forecasting electricity prices with input/output hidden Markov models, IEEE Trans. Power Sys. 20(1) (2005) $13-24$.

[7] A. Baíllo, M. Ventosa, A. Ramos and M. Rivier, Optimal offering strategies for generation companies operating in electricity spot markets, IEEE Trans. Power Sys. 19(2) (2004) 745-753.

[8] L. Bayón, J. Grau, M.M. Ruiz and P.M. SuÁrez, Nonsmooth Optimization of Hydrothermal Problems, Journal of Computational and Applied Mathematics 192(1) (2006) 11-19.

[9] F.H. Clarke, Optimization and Nonsmooth Analysis, SIAM, Philadelphia, 1990.

[10] L. Bayón, J. Grau, M.M. Ruiz and P.M. SuÁrez, New developments on equivalent thermal in hydrothermal optimization: an algorithm of approximation, Journal of Computational and Applied Mathematics 175(1) (2005) 63-75. 